\title{
e-Migrinter
}

$11 \mid 2013$

Et l'immobilité dans la circulation?

\section{Mobilité des enfants et des jeunes sous conditions d'immobilité ?}

\author{
Ce que circuler signifie
}

\section{Sandrine Depeau}

\section{Édition électronique}

URL : https://journals.openedition.org/e-migrinter/302

DOI : 10.4000/e-migrinter.302

ISSN : 1961-9685

\section{Éditeur}

UMR 7301 - Migrinter

\section{Édition imprimée}

Date de publication : 1 septembre 2013

Pagination : 103-115

ISSN : 1961-9685

\section{Référence électronique}

Sandrine Depeau, « Mobilité des enfants et des jeunes sous conditions d'immobilité ? », e-Migrinter [En ligne], 11 | 2013, mis en ligne le, consulté le 20 mai 2021. URL : http://journals.openedition.org/emigrinter/302 ; DOI : https://doi.org/10.4000/e-migrinter.302 


\section{(e-migrinter \\ Mobilité des enfants et des jeunes sous conditions d'immobilité ? Ce que circuler signifie}

Sandrine Depeau

L'ensemble des photographies et des documents ont été réalisés par l'auteur.

$\mathbf{L}$ a question de la mobilité des enfants est discutée ici sous l'angle de la dialectique « mobilité - immobilité " posée par ces journées d'étude. Un tel point de vue invite à considérer autrement la place, le rôle, les fonctions et les valeurs de la mobilité dans la société d'aujourd'hui. En effet, interroger les déplacements au regard de l'immobilité oblige à poser d'emblée la diversité des acceptions terminologiques et le sens des mobilités. Dans quel registre nous situons-nous ? Celui du spatial, celui du social, celui de

l'activité, celui des valeurs, celui des désirs ou des représentations, etc. ? Que peut révéler l'entrée par

l'immobilité sur le processus de mobilité des enfants?
Une première partie de mise en perspective empirique et théorique permettra de dégager le sens de chacun de ces registres en mettant en relief mobilité et immobilité. De là, en prenant appui sur quelques résultats de deux enquêtes réalisées sur les bus pédestres (pedibus) auprès de familles (parents et enfants d'âge primaire habitant l'agglomération rennaise), nous montrerons à partir de quatre grandes lignes de mise en tension des notions, comment la question de la mobilité des enfants est imbriquée dans un ensemble d'enjeux dissonants et qui pour autant permettent de saisir les dimensions et fonctions fondamentales de la mobilité au cours de l'enfance. Les entrées de discussion seront centrées sur: 1) le dispositif de bus pédestre dans ses dimensions sociales, spatiales et son potentiel éducatif ; 2) le rôle des parents (hypothèse de dissonance entre ancrage et mobilité) ; 3) le rôle des parents dans le rapport à la mobilité et enfin, 4) les valeurs normatives et sécuritaires de l'immobilité comme conditions de la mobilité des enfants.

\section{Mobilité dans les espaces immobiles}

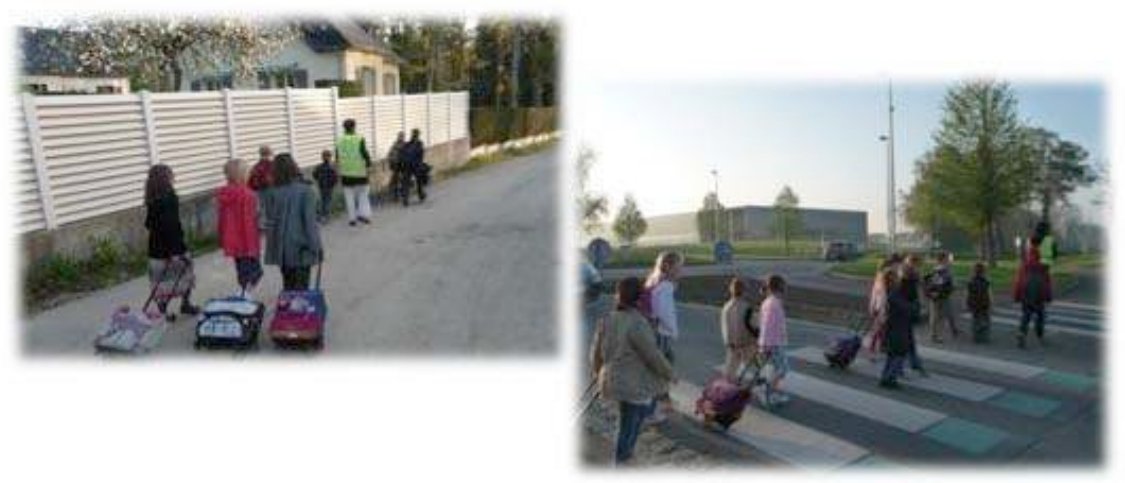




\section{De la mesure, aux situations et dispositifs}

\section{Perspectives empiriques et théoriques à l'appui}

Les enfants face à la mobilité : un constat de régression

Depuis plus d'une vingtaine d'années, la libre circulation des enfants dans l'espace urbain n'est plus une observation indépendante des déplacements des adultes. Subissant le développement de la ville automobile, les déplacements autonomes des enfants ont en effet connu une nette régression. Depuis peu, sous l'avènement de la ville pédestre, la mobilité des enfants évolue toutefois sous conditions d'immobilité, ou dit autrement s'apparente à des formes de mobilité canalisée.

L'étude qui fait référence dans le domaine est celle de Hillman et al. (1990) sur l'indépendance de déplacements des enfants en milieu urbain en Angleterre. Elle montre qu'en l'espace de dix ans, les modes de déplacements ont sensiblement évolué :

- les trajets faits seul ont diminué de $15 \%$, pour les enfants de $7-9$ ans, et beaucoup plus faiblement, pour les enfants de 11 ans ;

- aller à l'école à pied diminue de $20 \%$ environ, tandis que l'accompagnement en voiture augmente d'autant ;

- le fait d'être non accompagné pour aller à l'école a diminué de près de $40 \%$, chez les enfants âgés de 11 ans ;

- l'autorisation de traversée de route a en revanche peu diminué, chez les enfants de 11 ans.

D'autres études plus récentes confirment cette diminution de la mobilité des enfants (O’Brien et al., 2000 ; Kyttä, 1997; Mc Millan et al., 2006). Mais bien avant toutes ces études, il parait pertinent de rappeler celle de Gaster en 1991, qui montre à partir de cinq indicateurs mesurant l'usage de la ville par les enfants, que sur trois générations (de 1915 à 1976) les accès à la ville avaient diminué et que les activités des enfants étaient devenues de plus en plus casanières. Et ce bien avant que les jeux informatiques et vidéo envahissent l'espace domestique. Cette étude révèle alors que les premières permissions pour sortir se font plus tardivement et qu'au cours des trois générations, les lieux fréquentés par l'enfant sont de moins en moins divers. Enfin, elle interroge aussi la présence de l'adulte qui devient de plus en plus importante dans les activités de l'enfant en dehors du domicile, en particulier du fait de leur incessante institutionnalisation rendant la pratique urbaine de moins en moins informelle.

Cette étude rarement citée est d'autant plus marquante qu'elle renvoie, à partir de son exploration du concept de home-range (étendue du chez-soi) à tout un ensemble de mesures qui invite à interroger, sous un angle différent, certains indices relatifs aux enquêtes déplacements. En effet, la mobilité des enfants a aussi été, pendant longtemps, l'oubliée ou la négligée de ces grandes enquêtes déplacements, dont la direction première consistait à relever sur une période donnée les grands mouvements et les déplacements d'une longueur significative.

Appréhension des mobilités - immobilités dans les enquêtes

Enquête déplacements vs enquête budget-temps $\Rightarrow$ " effet de réponse molle ".

- La mobilité dans l'enquête budget-temps => activité supérieure à $10 \mathrm{~min}$.

- Mobilité de l'enfant => impensée des enquêtes ou confondue avec celle des adultes accompagnateurs.

- La mobilité restreinte des enfants est conditionnée par la mobilité automobile. 
Quand la norme des mesures néglige des mobilités singulières

Dans ce contexte, Hubert et al (2008) dans leur étude comparative relative aux enquêtes déplacements montrent comment les notions d'immobilité et de mobilité peuvent être entendues différemment selon qu'on se situe dans un cadre d'enquête de type déclaration de déplacements, ou bien dans un cadre de type budget-temps. La prise en compte d'un déplacement est liée à un temps minimum requis pour comptabiliser le trajet comme activité, soit dix minutes. Ce temps pris en compte n'aurait d'impact, non pas tant sur les codages de l'enquêteur, que sur la prise en compte de l'activité de déplacement court par les répondants eux-mêmes. La différence proviendrait de la manière de répondre à l'enquête déplacement, notamment l'effet de « réponse molle ».

Les déplacements piétons, qui plus est de courte distance, peuvent être négligés au point d'assimiler la mobilité à un mouvement borné par un seuil minimal d'étendue de déplacement (et donc de vitesse), excluant dans cette logique les enfants. Cette moindre importance des déplacements lents a été pour une grande part liée à l'objectif de cerner les grands mouvements motorisés pour façonner et corriger les territoires jusqu'à ce que le mode pédestre revienne en vogue. Dans ce contexte, les manières de mesurer la mobilité dans les enquêtes nationales ont infléchi en quelque sorte la définition de la mobilité. Dans certains cas, un déplacement inférieur à dix minutes n'est pas considéré comme une mobilité mais bascule du côté de l'immobilité. Elle serait de ce fait réduite à une forme impensée des enquêtes.

En même temps, on peut affirmer que cette quasi-immobilité déduite des enquêtes puis observée dans les études spécialisées sur l'enfance entretient, dans un contexte d'aménagement de fluidité urbaine, la circulation des autres. C'est du moins ce que l'on peut déduire des facteurs exposés de manière récurrente et dans les études sur l'enfance, qui se réduisent souvent à la densité d'un trafic automobile allant croissant.

\section{Statuts des mobilités enfantines Mobilité -immobilité}

- Mode (déplacement) $=>$ vitesse $=>$ distance

- Activité : objectif / destination

- Mouvement : défini relativement à l'arrêt (<10 min)

- Etat: autonome ou non (Accompagnement)

- Valeur

- Processus / dispositif
- $\quad$ Vitesse $=>$ lenteur

- Lieux : différenciés vs confondus (permanents)

- Mouvements $=>$ sédentarité

- Etat : indépendance $=>$ dépendance Etat : actif vs passif

- Valeur : positive vs négative

$=$ Processus $=>$ activité aux fonctions développementales (sociales et cognitives)

- expérience du corps

- rapport à l'autre

- rapport au temps

- rapport à l'espace et codes de circulation

$\Rightarrow$ forme de compétence de mobilité fondamentale

Mobilité proche de l'activité déployée dans les " hauts lieux " (Moncomble, 2009) 
Enfin, négligée parce que trop insignifiante du fait des distances parcourues mais aussi souvent confondue avec la mobilité des femmes, tout du moins souvent prise en compte comme facteur explicatif de la plus grande automobilité des femmes (Dupuy, 2000), la manière de considérer la mobilité des enfants dans les grandes enquêtes nationales a réduit pendant longtemps ces derniers au statut d'immobilisés, parce qu'escortés et donc invisibles dans l'activité de déplacement. Les normes de la mesure du déplacement, pour une grande part assimilées à celle du mode (et d'une certaine manière à la vitesse), donnent un premier registre de définition de la mobilité, entendue comme une activité, en même temps qu'une distance suffisante qu'on parcourt. À ce titre, il n'est pas risqué d'interpréter la mobilité des enfants comme une quasi-forme d'immobilité. De là, la part active du déplacement n'en est que davantage renforcée.

De la reconnaissance d'une mobilité régressive...

À ces mesures fondamentales, vient s'ajouter celle de la prise en compte de l'épaisseur du déplacement dans les espaces traversés et les activités réalisées au cours du déplacement. Cette épaisseur, considérée en tant que telle seulement depuis peu sous l'exigence des besoins de requalification urbaine des espaces de la marche entre autres, de compréhension et définition des espaces transactionnels (Moncomble, 2009) et de réorientation de la ville dense vers des stratégies de démotorisation, élargit les acceptions de la mobilité.

En redonnant une valeur à l'activité même du déplacement, à son caractère actif ou non, la définition de la mobilité pour une population telle que celle des enfants gagne un sens plus élargi, qui retient le mode d'accompagnement mais surtout la notion d'indépendance et/ou de libre choix. Ainsi, dans le champ d'étude de l'enfance, être mobile revêt un sens différent de la définition donnée par les enquêtes classiques. Là où les dimensions de vitesse (temps d'accès) et donc de modes suffiraient à circonscrire la définition de la mobilité, ici ce sont plutôt les dimensions d'activité et d'indépendance (dans l'accompagnement) qui justifient la définition, et plus largement, engagent les recherches sur les effets sociaux et cognitifs d'une régression de l'autonomie de déplacements des enfants (Depeau, Ramadier, 2005).

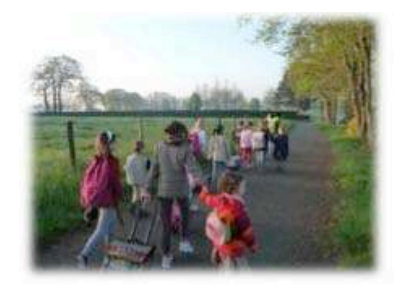

Dispositif : cadre normatif important
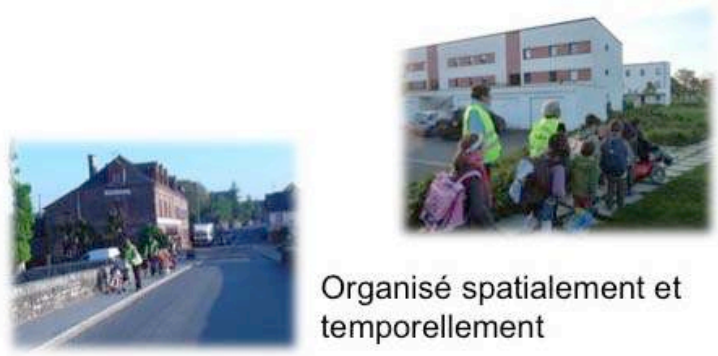

Organisé spatialement et temporellement

Pedibus ou bus pédestre : dispositif institutionnalisé dans le cadre des plans de déplacement école qui consiste en un groupe d'enfants accompagnés par deux ou trois adultes sur les trajets scolaires quotidiens. 
Davantage encore, c'est l'expérience du corps dans l'espace, dans ses limites, ses contraintes et libertés qui donnent sens à la notion de mobilité. Et ce, dans une définition qui se rapprocherait finalement de celle profilée dans le registre de «l'hommemasse » (Mocomble, 2009) utilisé pour décrire l'urbanité impensée de nos sociétés des hauts lieux. Autrement dit, un ensemble de déplacements pris dans une mixité sociale expansive caractéristique des lieux de transit qui impose dans la logique des flux «un corps-à-corps qui se constitue en médium des conduites et des valeurs soumises aux prescriptions des réseaux et des flux » (Moncomble, 2009 : 382). Ce rapport à la mobilité s'inscrit dans des milieux plutôt denses et caractéristiques des lieux de l'intermodalité. Pour autant, il paraît intéressant pour comprendre les logiques sous-jacentes à la mobilité des enfants. D'une part parce qu'il permet de mettre en tension sous une forme particulière les domaines de la sédentarité et du mouvement, et tout ce que cela suggère dans les rapports au temps, aux espaces et aux autres. Et d'autre part, parce qu'une fois posée cette dialectique, il permet d'inscrire le déplacement des enfants d'une problématique familiale œuvrant dans une dualité mobilité vs immobilité, désir de mouvements vs désir d'ancrage, etc.

\section{... à la constitution d'une mobilité canalisée}

Depuis une dizaine d'années, sous l'injonction du développement durable et le renouveau des villes pédestres, la mobilité des enfants évolue, régie entre autres par la mobilité scolaire. Laquelle, en tant que composante des indicateurs d'autonomie de déplacement des enfants, tend à être de plus en plus formatée en offrant de nouveaux cadres normatifs. Les bus pédestres (ou pedibus) composent ceux-ci. Ils se définissent comme une caravane d'enfants accompagnés d'adultes pour le trajet scolaire et sont des vecteurs importants de promotion des mobilités alternatives à l'automobilité. Ce type de mobilité inscrite dans des formes de récurrence spatiale et temporelle devient dès lors fondamental. En effet, parallèlement aux questions de bienêtre et de sécurité propres aux valeurs d'ancrage, celle des déplacements scolaires constitue un des curseurs de la gestion du trafic intra-communal (notamment la congestion automobile aux abords des écoles) et plus encore, de la valorisation et de la gestion durable des mobilités «douces» quotidiennes dans la politique urbaine. En tant que déplacements spatialement, socialement et temporellement contrôlés, les bus pédestres constituent des dispositifs de circulation qui questionnent la mobilité des enfants de façon plus générale. Ils ouvrent la voie à de nouvelles interrogations sur les rapports à l'espace et surtout sur le rapport mobilité-immobilité au sein du système familial.

\section{Quelques données d'enquêtes}

Un ensemble de données tirées de deux enquêtes réalisées dans l'agglomération rennaise, auprès d'enfants et de leurs parents interrogés individuellement constitue le matériau de base pour étayer la discussion autour de quatre lignes directrices. Ces deux enquêtes visaient à comprendre les fonctions sociales, cognitives, éducatives et identitaires des bus pédestres.

Une première enquête par entretiens semi-directifs et passation de Jeu de reconstruction spatiale (Ramadier, Broner, 2006) a été réalisée à Cleunay, quartier au Sud-Ouest de la ville de Rennes qui fait partie d'un des cinq quartiers d'expérimentation de pédibus de la ville. Dans le quartier de Cleunay, deux lignes de pedibus (" ligne rouge » et « ligne verte ») étaient rattachées à l'époque de l'enquête à un même groupe scolaire à partir duquel a été sélectionnée la population interrogée. 
Enquête 1 : familles impliquées vs non impliquées dans le pedibus

- Terrains : Quartier au Sud-Ouest de la ville de Rennes, il fait partie d'un des cinq quartiers d'expérimentation de pédibus de la ville. Deux lignes de pedibus vers une seule école publique.

- Entretiens semi-directifs et passation du Jeu de Reconstruction Spatiale (JRS) auprès d'enfants et parents rencontrés individuellement.

- Deux groupes de familles: impliqués vs non impliqués.

\section{Enquête 2 : dispositifs dans l'agglomération rennaise}

- Quatre communes de l'agglomération rennaise.

- Campagne d'entretiens semi-directifs.

- Deux échantillons de parents $(n=52)$ et d' enfants $(n=40)$ interrogés au domicile individuellement.

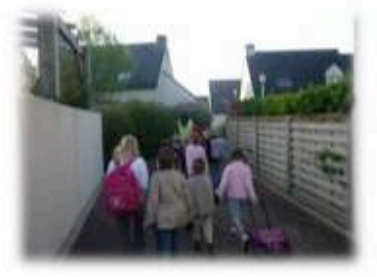

Deux groupes de familles (enfants et parents) selon qu'ils sont impliqués ou non dans un pedibus ont été rencontrés et interrogés lors d'une enquête par entretiens semi-directifs individuels : 1) un échantillon total de 30 enfants, 15 impliqués dans le pédibus vs 15 non impliqués (moyenne d'âge : 8,8 ans pour les enfants impliqués; moyenne d'âge : 9,6 ans pour les enfants non impliqués); 2) un second échantillon, constitué de parents répartis selon la même condition que pour les enfants $(n=26)$ a été interrogé au domicile principalement.

La seconde enquête a été réalisée sous la forme d'une campagne d'entretiens plus approfondis menés avec tous les acteurs des bus pédestres, parents, enfants et accompagnateurs non parents. La campagne d'enquêtes s'est concentrée sur quatre communes choisies sur des critères précis extraits du rapport de bilan des pedibus de 2009 réalisé par le Comité Local à l'Environnement (CLE) de l'agglomération rennaise. Deux échantillons de parents $(\mathrm{n}=52)$ et d'enfants $(\mathrm{n}=40)$ ont été interrogés au domicile individuellement à partir d'entretiens semi-directifs. Par ailleurs, six lignes de bus pédestres dans les quatre communes sélectionnées ont été suivies et observées in situ par deux enquêteurs.

Le dispositif de bus pédestre: une mobilité canalisée

Les données d'enquêtes mobilisées reposent sur: les observations réalisées in situ lors de suivis de bus pédestres; les raisons de s'impliquer dans le dispositif; l'évaluation du dispositif par les enfants; les données liant autonomie scolaire et déplacement extrascolaires.

Inscrit dans une trame spatiale et temporelle récurrente, le bus pédestre engage les enfants dans une mobilité fortement canalisée. D'un point de vue spatial, ils suivent des trajets soigneusement étudiés, souvent immergés dans les coulées vertes des lotissements et éloignés, quand l'itinéraire le permet, des points importants de circulation permettant d'assurer la sécurité des déplacements. Pour autant, ce balisage interroge les occasions de déplacements buissonniers, de détours, de raccourcis qui constituent par ailleurs des 
expériences importantes d'apprentissage du déplacement. D'un point de vue temporel, les trajets sont calés sur des horaires précis et sur un rythme de marche souvent imposé par les plus jeunes enfants (la limite d'âge inférieure est de 4 ans). Enfin, d'un point de vue social l'encadrement et l'interventionnisme des adultes en font un trajet sous contrôle constant. C'est par ailleurs, un dispositif à forte homogénéité sociale: les parents impliqués sont issus de catégories sociales supérieures ou intermédiaires, disposant d'une certaine flexibilité temporelle. Ce sont également des familles très impliquées dans l'éducation ainsi que dans d'autres formes associatives.
Par ailleurs, les formes de cheminements programmés minimisent, en dehors du groupe lui-même, les occasions d'interactions sociales, la rencontre de l'autre et la complexité urbaine. Laquelle suppose que s'entrelacent l'individuel et le collectif, le routinier et l'inattendu, le surprenant et le rassurant. Partant, on pourrait même dire qu'il s'apparente, au vu des comportements des enfants, à une forme de trajet « embullé » (Depeau, 2008). En effet, le bus pédestre dans l'apprentissage de l'espace urbain pourrait être associé à une bulle mobile non seulement dans les faits, mais également dans les effets en termes de développement cognitif et de développement de l'autonomie.

\section{Un dispositif spatialement et temporellement canalisé}

Un cheminement :

- en zone verte (distance du trafic recherchée)

- évitant les ruptures de trajet

Un rythme impulsé :

- par la cadence de marche des plus jeunes enfants

- par les arrêts de ramassage
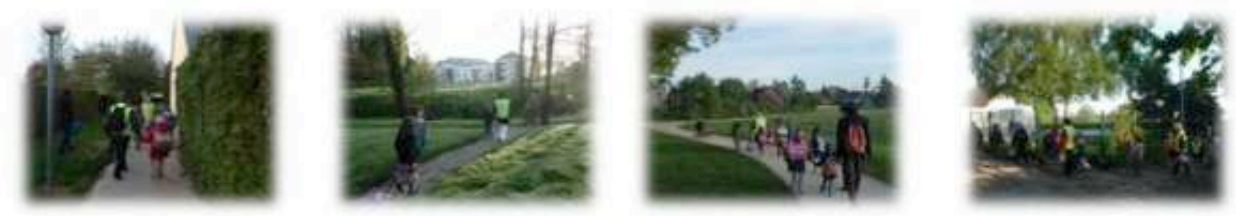

Un dispositif socialement marqué

Un groupe de parents socialement homogène

- Catégories sociales supérieures, intermédiaires

- Catégories disposant de faibles contraintes horaires

- Des familles très impliquées dans l'éducation

- Adhésion associative fréquente 


\section{De l'assurance du familier au besoin d'indépendance}

Evaluation des enfants:

- Lassitude des enfants (en désir d'autonomie)

- Besoin de sortir des traces du groupe

- Besoin d'avoir son propre rythme

- Besoin d'expérimenter des chemins buissonniers

- Besoin de suivre d'autres groupes (amis)

Les résultats de la deuxième enquête montrent que le dispositif n'est que rarement le tremplin d'une autonomie extra-scolaire. On remarque en effet que les traces du dispositif sont très peu utilisées comme une situation d'amorce à l'autonomie. Quelques enfants font le retour de l'école seuls ou avec des pairs; un tiers rentre encore en voiture de l'école; certains sont autorisés à un trajet autonome pour quelques courses, d'autres utilisent le tracé ou parcours du pedibus pour aller à des activités, cependant ces expériences ne concernent qu'une très faible proportion d'enfants. On observe d'ailleurs à l'âge d'entrée en autonomie, une lassitude des enfants, un besoin de sortir des traces du groupe, de trouver son propre rythme, de découvrir d'autres chemins et de suivre d'autres groupes (les amis, par exemple).

Lors de la première enquête, les résultats reflétaient déjà la même tendance. La comparaison des scores moyens entre parents des deux groupes met en évidence une évaluation de l'autonomie légèrement plus élevée dans les familles non impliquées dans le pédibus que dans celles impliquées, autant dans les réponses des parents que dans celle des enfants.

Lors de la deuxième série d'enquêtes, un indice d'autonomie a été calculé pour chaque enfant, afin de résumer l'ensemble des pratiques autonomes dans ses différentes modalités, en termes de déplacement et d'accompagnement. Un poids à valeur croissante est donné à chaque configuration de déplacement. Ainsi, plus l'enfant utilise un mode actif de manière indépendante (sans la présence d'un adulte, ou d'un pair), plus l'indice pondéral est élevé et plus le score final (rapport des configurations sur l'ensemble des activités) est important également. Pour l'ensemble des enfants interrogés, l'autonomie de déplacement ne semble que peu ancrée dans les pratiques. En moyenne, le score d'autonomie est inférieur à 3. Ce qui représente un niveau d'indépendance et de déplacement actif assez faible (la valeur maximale potentielle oscillant autour de $8-9$ ). Mais l'observation des moyennes selon la commune de résidence des enfants révèle quelques nuances.

\section{Faible effet d'encouragement aux déplacements autonomes}

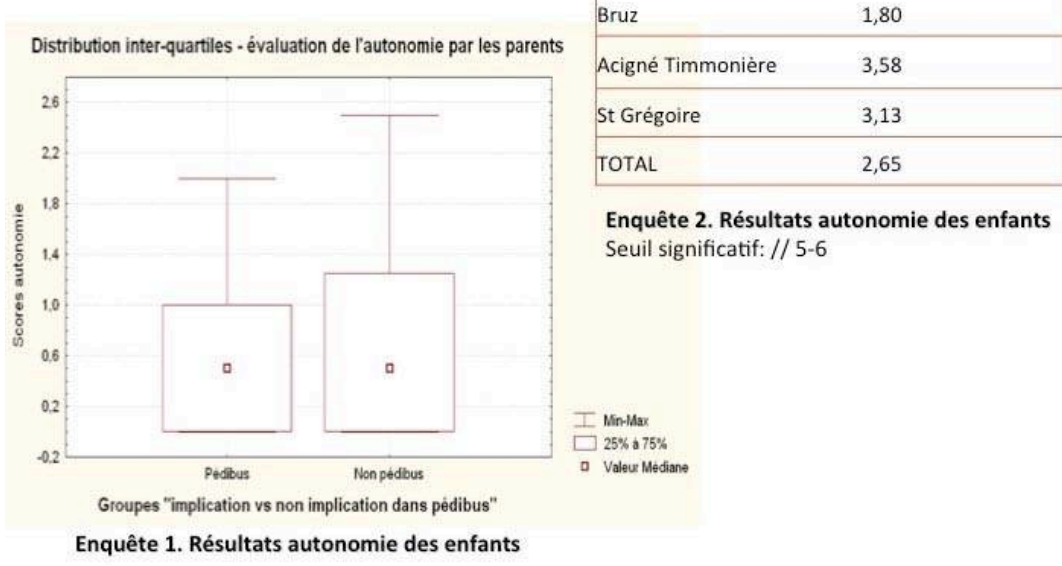

\begin{tabular}{|ll|}
\hline Communes enquêtées & Coef. Autonomie \\
\hline Le Rheu & 1,17 \\
\hline Bruz & 1,80 \\
\hline Acigné Timmonière & 3,58 \\
\hline St Grégoire & 3,13 \\
\hline TOTAL & 2,65 \\
\hline
\end{tabular}

Enquête 2. Résultats autonomie des enfants

euil significatif: // 5-6 
La mobilité des enfants comme fonction révélatrice des rapports dissonants entre ancrage et mobilité des familles

Les données d'enquête mobilisées concernent ici: les choix résidentiels, l'évaluation du quartier de résidence, les raisons d'une implication dans les alternatives de mobilité "douce », les choix de lieux pour l'enfant. La question des déplacements des enfants, et en particulier de l'apprentissage de l'autonomie, marque un stade du développement de l'enfant en même temps qu'un événement fondamental dans le cycle de vie familiale qui vient remettre en question l'ensemble des rapports à l'espace de la famille, et qui génère de nombreux remaniements sociaux et spatiaux des parents. C'est donc tout l'équilibre entre des attentes liées à l'éducation des enfants et d'autres relatives aux qualités de l'espace résidentiel qui est remis en question au point d'engendrer certaines dissonances cognitives.

Pour certains auteurs, l'attachement au lieu, associé à la notion de mobilité, est prédictif d'un assignement au lieu pour les classes les plus défavorisées ayant de faibles capacités de déplacement (Bauman, 1998). Dans certaines situations néanmoins, dans certaines phases de transition (voire certaines phases critiques) du cycle de vie d'un individu ou d'un groupe, l'attachement au lieu peut avoir une fonction compensatrice (Hay, 1998) en lien avec la mobilité qui permet d'assurer la continuité des liens, l'équilibre, tout en renforçant ou recomposant des façons de se singulariser.

\section{Liens entre mobilités et désir d'ancrage}

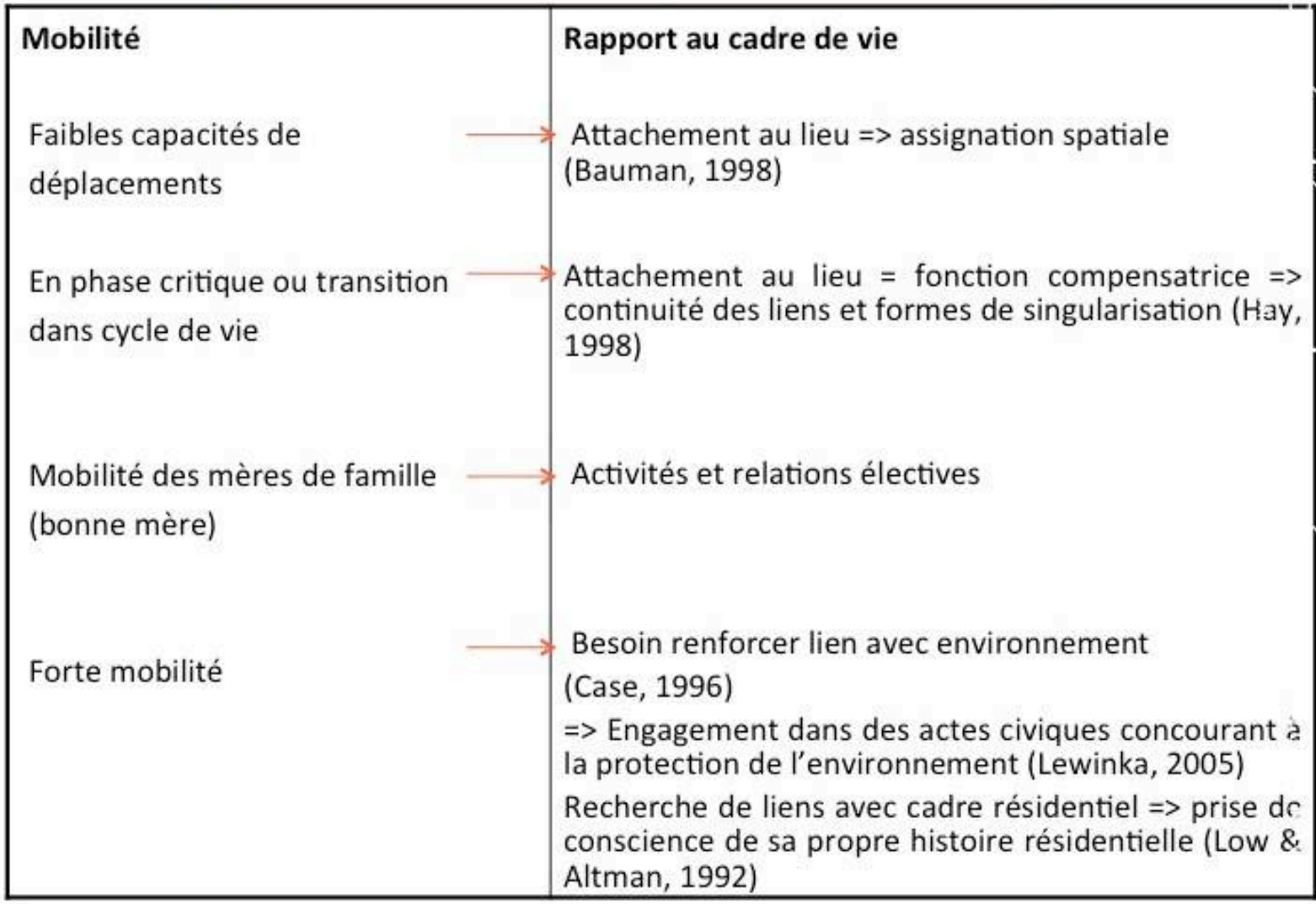


Dans d'autres cas, la mobilité provoque le besoin de renforcer des liens avec l'environnement immédiat (Case, 1996), le lieu-centre où s'inscrit la sédentarité. L'attachement au lieu ne serait pas seulement la conséquence d'une assignation géographique forte liée à une faible mobilité, et donc à des groupes à faible capital culturel et économique, mais la conséquence de nombreux déplacements réalisés hors quartier de résidence. Ces liens peuvent être activés de différentes manières. Très souvent associés aux liens sociaux et à différentes formes de sociabilité dans le quartier, ils peuvent également inspirer toute forme d'engagement dans des actes civiques, bénévoles concourant à la préservation de l'environnement résidentiel (Lewicka, 2005). Identité et actes civiques ne seraient pas seulement le fait de groupes peu mobiles, mais aussi de groupes cherchant à ressouder les liens distendus par leurs différents déplacements qui mettent à mal l'identité résidentielle.

Dans le cadre des enquêtes réalisées, on a donc posé l'hypothèse que l'investissement dans le bus pédestre agirait comme une forme de réaffirmation de soi, un mode de rationalisation de ses comportements journaliers, une résipiscence face aux comportements du quotidien. Autrement dit, s'impliquer dans un tel dispositif signifierait implicitement s'affirmer dans le jeu des positions d'habitants en même temps que dans celui des positions libérales et environnementales. Par ailleurs, la pratique du pedibus pourrait avoir un rôle de distinction d'autant plus fort que leur publicisation et leur médiatisation participent à ces formes de normalisation de comportements dits «soutenables» et donc "désirables », comme pour contrecarrer une image négative de la périphérie urbaine, trop souvent assignée à une «mobilité débridée ».
Stratégie éducative qui permet non seulement de contrôler le contexte spatial et social des déplacements des enfants, mais également de renforcer certains attachements au quartier

La mobilité des enfants s'inscrit dans des questions plus générales relatives aux stratégies résidentielles et aux stratégies éducatives. Elle peut dès lors constituer une des modalités du processus d'identité spatiale des parents. Dans cet objectif, les questions de quartier idéal pour la famille et pour l'enfant, d'attachement au milieu résidentiel, de sentiment d'appartenance à la commune de résidence ou encore à une communauté scolaire particulière, permettent de repérer quelques enjeux identitaires liés à l'implication dans le dispositif de mobilité.

Les résultats des deux enquêtes montrent que la notion de quartier idéal renvoie pour les parents à un contexte qui permet de préserver un cadre de stabilité (et donc de sédentarité) pour l'enfant. L'accessibilité des espaces repose, pour plus d'un parent sur deux, sur la proximité de l'école et des autres espaces de loisirs, et pour plus des deux-tiers, sur la sécurité des déplacements $(69 \%)$. La proximité du réseau social des enfants minimisant les déplacements est une autre dimension associée au quartier idéal. Le besoin de créer un cadre proche des formes du village et les relations interpersonnelles qui le caractérisent sont fréquemment mis en avant. Près des deux-tiers des parents se disent attachés au quartier au point d'imaginer l'idée de déménagement comme un déracinement, notamment pour les enfants dont ils cherchent à préserver un niveau de sociabilité.

Par ailleurs, dans un contexte perçu comme agréable, près d'un quart des parents se disent gênés par les contraintes de circulation automobile. Le pedibus apparait alors comme un moyen «d'éviter les déplacements voiture, un gain de temps, une façon de désencombrer le parking de l'école, 
une manière d'être à l'heure pour l'enfant (...) mais aussi une bonne réponse à l'immobilisme de l'enfant qui permet de le responsabiliser ». Mais en guise de valorisation, le discours de certains parents révèle parfois à quel point le mode motorisé est ancré dans des routines. Pour quelques parents, le pedibus c'est en effet « aller à pied à l'ancienne ».

Le dispositif pourrait être assimilé à un espace interstitiel œuvrant comme une parenthèse de respiration pour la famille et chacun de ses membres. Il permettrait aux parents de s'affranchir des contraintes sociales et temporelles, pour aider l'enfant à franchir les frontières fonctionnelles et spatiales.

Le désir d'ancrage et de confort pour les enfants dans l'espace résidentiel qui dans le système familial s'apparente à un désir d'immobilité de l'enfant.

La mise en parallèle des données de mobilités scolaires et extrascolaires permet d'expliquer une faible autonomie des enfants en dehors des trajets scolaires. Cette dernière révèle aussi un taux de navettes important des mères de famille. Les trois-quarts des enfants interrogés (enquête 2) sont accompagnés par la mère de famille aux activités extra-scolaires. Les navettes d'accompagnement des enfants pour ces activités seraient entendues comme des moyens de maintenir, outre le confort de l'enfant, l'ordre sédentaire des modes de vie dans l'espace résidentiel à travers notamment la maitrise des distances à l'autre.

L'exigence d'une stabilité des cadres d'activités pour l'enfant de même que la recherche d'une homogénéité sociale de ces cadres entraîne une plus grande mobilité des parents et plus spécifiquement des mères de famille. Ces mères qui circulent en limitant le potentiel d'autonomie des enfants ont aussi la maîtrise des lieux fréquentés par ces derniers. Dans le cadre de remaniements identitaires liés aux transformations familiales, la mobilité joue parfois un rôle fondamental au point de générer des styles de vie autrement appelés «cultures» par certains auteurs comme Dowling (2000). Robyn Dowling montre comment, de l'utilisation de la voiture pour l'accompagnement des enfants, aux choix de lieux pour leurs activités extra-scolaires, certaines femmes parviennent à renforcer leur identité de "bonne mère » à travers le jeu de rôles activé au cours de routines éducatives quotidiennes. Dans cette perspective, la mobilité des mères permet non seulement de renforcer l'identité et les rôles qui lui sont assignés, mais également de stabiliser le cadre et les repères de l'enfant pour maintenir des formes de continuité des cadres sociaux.

En dehors des temps scolaires, les lieux autorisés aux enfants en âge de se déplacer seuls sont très circonscrits spatialement et sont également ceux qui assurent un cadrage social et temporel (i.e. le réseau amical de l'enfant et de la famille). La mobilité des enfants dans la représentation des mères de famille a valeur de distance au lieu connu. Les négociations en termes d'autorisation s'inscrivent d'ailleurs dans ces questions de distances spatiales mais aussi sociales.

\section{Les valeurs normatives et sécuritaires de l'immobilité comme conditions de la mobilité des enfants}

$\mathrm{Si}$ les négociations en termes d'autorisation s'inscrivent dans la question de la distance au lieu connu, elles renvoient aussi à des formes plus normatives d'usage des espaces extérieurs. Être en mouvement, éviter les arrêts sont quelques leitmotivs repérés dans les recommandations parentales. Par ailleurs, si le déplacement de l'enfant se résume toujours plus à une circulation sans arrêts, notons également que les lieux autorisés à l'enfant dans le cadre de déplacements autonomes sont très fréquemment, voire exclusivement, des lieux liés au réseau social et familial, comme le 
domicile des amis, le domicile d'un membre de la famille, voire encore un lieu d'activités où le parent est assuré d'un contact potentiel.

Le besoin de connaissance et d'assurance de point fixes pour l'accueil des enfants (repérés dans le discours des parents) conditionne la mobilité des enfants. La présence de lieux de type refuge à forte familiarité sociale augmente la réassurance des parents. En outre, cette mobilité autonome est conditionnée par le besoin d'une garantie de la fluidité du trajet de l'enfant, dit autrement par le fait que l'enfant respecte un temps de déplacement, qu'il ne "traine pas ", voire ne stationne pas trop longtemps. Il y aurait des lieux plus propices à l'arrêt que d'autres, régis par une double injonction consistant à interdire le stationnement dans les espaces publics et à limiter les circulations. Cette double injonction participe d'ailleurs de la norme sociale et individuelle qui tend à marginaliser par la même occasion ceux qui continuent à séjourner dans les espaces publics (Valentine, 1996). Cette marginalisation trouve quelques origines dans les normes qui façonnent l'ordre public en termes d'usage des espaces qu'il s'agisse du stationnement ou de la circulation.

Dans cette perspective, une étude ethnographique des opérations de contrôle de services de surveillance par vidéo des espaces publics (Neyland, 2006) montre comment l'attente de mobilité, par les agents chargés de la surveillance vidéo d'espaces publics, façonne l'interprétation d'une série d'images vidéo d'enfants stationnant sans rien faire dans l'espace public. L'objectif de ces surveillances construit sur une série d'inférences évaluatives des comportements d'enfants et de jeunes vise à repérer ceux dits «à risques» (de délinquance) en vue de déclencher les opérations de contrôle en actes des enfants visionnés en amont. L'étude montre en somme comment les normes qui façonnent l'ordre public sont ancrées dans un système de réseaux d'acteurs qui œuvrent selon des opérations d'évaluation simplificatrices ancrées dans une dialectique mobilité-immobilité. Cette base d'inférences agirait dans un «circuit fermé », où les évaluations et attentes liées au stationnement (et donc à l'immobilité) sont très fortement dépendantes des lieux de stationnement et des formes de mobilités attendues par rapport à ce lieu. Par exemple, le stationnement à un arrêt de bus n'a pas le même potentiel d'inférence qu'un point non connecté à la mobilité, de même qu'une immobilité devant un restaurant.

Finalement, cette double injonction consistant à interdire le stationnement dans les espaces publics et à limiter les circulations, s'installe comme une norme sociale et individuelle qui, sans être propre aux enfants, n'en est que plus exacerbée.

Sandrine Depeau

CR CNRS

UMR 6590 ESO

sandrine.depeau@uhb.fr 


\section{Bibliographie}

Bauman, Zygmunt (1998) On globalization : Or globalization for some, localization for others, Thesis Eleven, n ${ }^{\circ 4}$, pp. 37-49.

Case, Duncan (1996) Contributions of journeys away to the definition of home : an empirical study of a dialectical process, Journal of Environmental Psychology, n¹6, pp. 115.

Depeau, Sandrine ; Ramadier, Thierry (2005) Les trajets Domicile-Ecole en milieux urbains: Quelles conditions pour l'autonomie de l'enfant de 10-12 ans?, Psychologie \& société, n³, pp. 81-112.

Depeau, Sandrine (2008) Nouvelles façons de se déplacer vers l'école ou l'expérimentation du pédibus dans un quartier rennais. Quelles incidences sur l'apprentissage de l'autonomie de déplacement des enfants et leurs rapports à l'espace ?, Recherche Transport Sécurité, n¹01, pp. 253-271.

Dowling, Robyn (2000) Cultures of mothering and car use in suburban Sydney: a preliminary investigation, Geoforum, $\mathrm{n}^{\circ} 31$, pp. 345-353.

Dupuy, Gabriel (2000) Automobilités : quelles relations à l'espace, in Bonnet, M. ; Desjeux, D. (eds) Les territoires de la mobilité, Paris, Presses Universitaires de France, pp. 37-51.

Gaster, Sanford (1991) Urban children's access to their neighbourhood: changes over three generations, Environment and Behavior, $\mathrm{n}^{\circ} 23$, pp. $70-85$.

Hay, Robert (1998) Sense of place in developmental context, Journal of Environmental Psychology, n¹8, pp. 5-29.

Hillman, Mayer ; John, Adams ; Whitelegg, John (1990) One False Move... A Study of children's independant mobility, London, PSI, $195 \mathrm{p}$.
Hubert, Jean-Paul ; Armoogum, Jimmy ; Axhausen, Kay; Madre, Jean-Loup (2008) Immobility and Mobility Seen Through Trip-Based Versus Time-Use Surveys, Transport review, n²8, pp. 641-658.

Kytta, Marketta (1997) Children's independent mobility in urban, small town and rural environments, in Camstra, R. (ed.) Growing up in a Changing Urban Landscape, Assen, Van Gorcum, pp. 41-52.

Lewicka, Maria (2005) Ways to make people active: The role of place attachment, cultural capital, and neighbourhood ties, Journal of Environmental Psychology, n²5, pp. 381-395.

McMillan, Tracy; Day, Kristen; Boarnet, Marlon ; Alfonzo, Mariela ; Anderson, Craig (2006) Johnny walks to school- does Jane? Sex differences in children's active travel to school, Children, Youth and Environments, $\mathrm{n}^{\circ} 16$, pp. 75-89.

Moncomble, Françoise (2009) Mobilités, réseaux, flux, in Stébé, J.-M. ; Marchal, H. (eds.) Traité sur la ville, Paris, Presses Universitaires de France, pp. 353-397.

Neyland, Daniel (2006) Moving images : the mobility and immobility of 'kids standing still', The Sociological Review, n54, pp. 363381.

O’Brien, Margaret ; Jones, Deborah ; Sloan, David ; Rustin, Michael (2000) Children's independent spatial mobility in the urban public realm, Childhood, $\mathrm{n}^{\circ}$, pp. 257-277.

Ramadier, Thierry ; Bronner, Anne-Christine (2006) Knowledge of the environment and spatial cognition: JRS as a technique for improving comparisons between social groups, Environment and Planning B : Planning and Design, vol. 33, n², pp. 285-299.

Valentine, Gill (1996) Children should be seen and not heard: the production and transgression of adults' public space, Urban Geography, n¹7, pp. 205-220. 